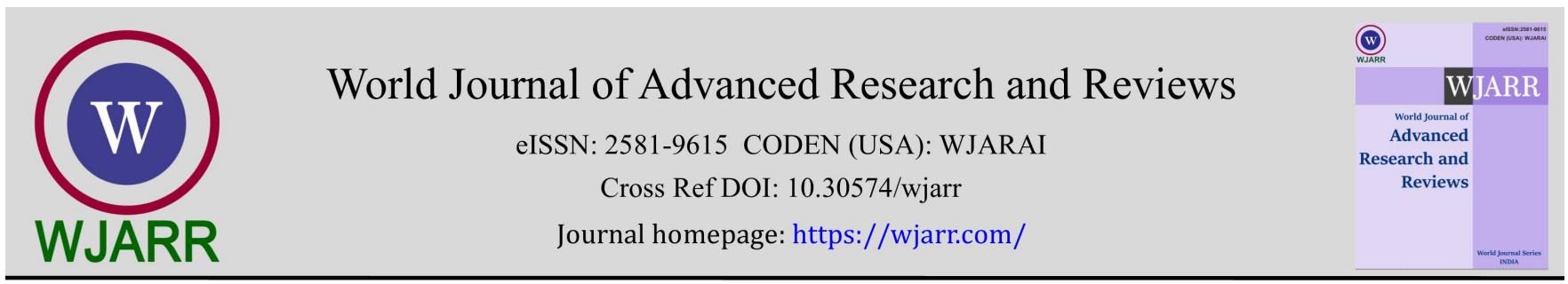

(REVIEW ARTICLE)

Check for updates

\title{
Focus on the totally laparoscopic feeding gastrostomy tube placement operative technique
}

\author{
Sorin Cimpean ${ }^{1, *}$, Alexandre Grapotte ${ }^{2}$, Nicolas Boyer ${ }^{1}$, Mathilde Poras ${ }^{1}$, Dario Raglione ${ }^{1}$ and Gloire à \\ Dieu Byabene 3
}

${ }^{1}$ Saint Pierre University Hospital Rue Haute, 322, 1000, Brussels
${ }^{2}$ Saint Luc University Hospital Avenue Hippocrate 10, 1200 Bruxelles
${ }^{3}$ Bukavu Hospital, Congo Avenue Michombero, Congo - Kinshasa.

World Journal of Advanced Research and Reviews, 2021, 09(01), 127-133

Publication history: Received on 02 January 2021; revised on 08 January 2021; accepted on 10 January 2021

Article DOI: https://doi.org/10.30574/wjarr.2021.9.1.0005

\begin{abstract}
Laparoscopic feeding gastrostomy placement is a surgical operation that allows the feeding of malnourished patients through a tube that is placed in the gastric lumen. The benefits of an improved nutritional status in terms of improving clinical outcomes are well documented in the literature and consist in a reduction of the complication rates of the surgical patients, the length of hospital stay, the readmission rates, and a reduction of the cost of health services by reducing the morbidity or mortality. We present a totally laparoscopic technique of feeding tube placement.
\end{abstract}

Keywords: Feeding Gastrostomy, Laparoscopy, Operative Technique

\section{Introduction}

Once with the early development of modern surgery the need for an alternative ways for feeding the patient who were not able to have an oral intake. The first notion of gastrostomy arise in 1839 when Sedillott performed gastrostomies in dogs. In 1876, Verneoil describe the first successful gastrostomy in humans in an early technique. Witzel in 1891 proposed the creation of a serosa tunnelling of the tube, this technique is mostly used for jejunostomy placement. Stamm in 1894 placed a purse-string suture to invaginate the tube into the stomach marked the most used technique of this intervention. 1 Gauderer, in 1980, described the first endoscopic approach for gastrostomy. The percutaneous approach is nowadays the gold standard for long enteral feeding and surgical gastrostomy it is used for specific indications. 2 Surgical gastrostomy, performed by laparotomy or by laparoscopy is indicated in case of head and neck malignancy with locally advanced cancer like stricture of the pharynx or the oesophagus who blocks the passage of the endoscope or lack of parietal transillumination, or any situations where the endoscopy is unavailable or impossible. 3

There are several techniques of gastrostomy, but we use a modified Stamm technique for the totally laparoscopic approach that we described here.

\footnotetext{
* Corresponding author: Sorin Cimpean

Saint Pierre University Hospital Rue Haute, 322, 1000, Brussels.

Copyright (@ 2021 Author(s) retain the copyright of this article. This article is published under the terms of the Creative Commons Attribution Liscense 4.0.
} 


\section{Operative technique}

\subsection{General principles}

The patient is placed in supine position on the operating table under general anaesthesia and oro-tracheal intubation. The surgical team is placed on the right side of the patient and the operative screen and the laparoscopy tour on the left side.

Antibiotherapy by third generation cephalosporin is indicated as the gastrotomy is performed and the potential contamination of the peritoneal cavity.

The trocar placement it's on the left flank: two $5 \mathrm{~mm}$ trocars for the instruments and one of $10 \mathrm{~mm}$ for the optical system.

\subsubsection{Instruments}

- $\quad$ Standard kit of laparoscopic surgery

- $1 \mathrm{~mm} \times 10 \mathrm{~mm}$ trocar

- $2 \mathrm{~mm} \times 5 \mathrm{~mm}$ trocars

- Kit of jejunostomy

- $\quad$ Suture threads

\section{Pneumoperitoneum creation}

The pneumoperitoneum created by the "open Hasson" or "modified Hasson" techniques or using the Veress or Palmer needle. The "open" technique is the safest and allows a quick intraabdominal entry of the first trocar but in case of obese patients the approach can be challenging due to the size of the abdominal wall. The main risk of the technique is the misidentification of the peritoneal sheet during the parietal dissection and perform a visceral injury. In case of multioperated abdomen these technique should be the technique of choice. 4 For the placement of the needle there are two usual localisations. The first is on through the ombilical scar, where the peritoneal layer is intimately fixed to anterior parietal wall and the trajectory is the shortest. The other localisation is in the left hypochondrium $1 \mathrm{~cm}$ under the costal grill on the anterior midaxillary line, where the viscera are more distantly and the risk of injuries are less. For this localisation the stomach must be well aspirated by the anaesthesiologist using a naso-gastric tube to avoid the intragastric placement of the needle. We preferred to place the Veress needle in the left hypochondrium because of the higher risk of visceral but mostly vascular vessels. The vascular injuries of the cava vein or of the aorta artery can put the patient's life in danger very rapidly.

\section{Trocar placement}

The first trocar of $10 \mathrm{~mm}$ is placed in the right flank. The periumbilical position is not suitable due to the proximity with the gastrostomy site and the difficulties of exposition. Once the optical trocar is positioned the $5 \mathrm{~mm}$ trocars are consecutively placed in the right hypochondrium and the right iliac fossae or periumbilical depending of the size of the patient abdomen and the proximity with the stomach. (Figure 1) It must be avoided the placement of the optical trocar and the right inferior trocar on the same axis with the stomach to not have a conflict between the instruments. Is imperative that the trocars to be placed under the visual control, to avoid visceral injuries or vascular injuries of the parietal vessels. Before placing the skin incision, if possible to perform a parietal transillumination who can help in identification of the parietal main vascular vessels. 


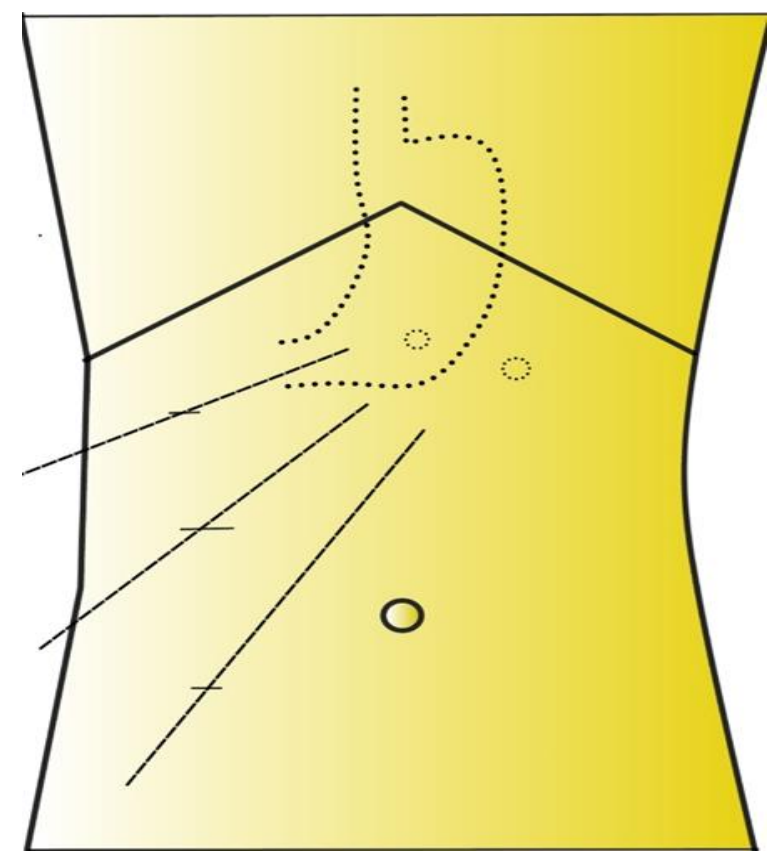

Figure 1 Trocar placement.

\section{Gastrotomy site}

The site of the gastrostomy is carefully chosen on the greater curvature of the stomach. The site of gastrotomy must not be too close to the greater omentum to avoid the vessels, at least $2 \mathrm{~cm}$. (Figure 2,3)

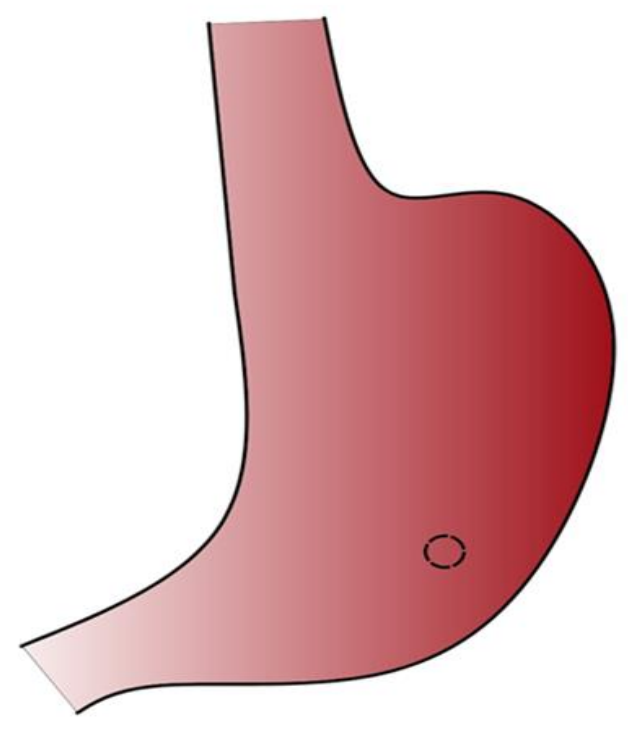

Figure 2 Choice of the gastrostomy site. 


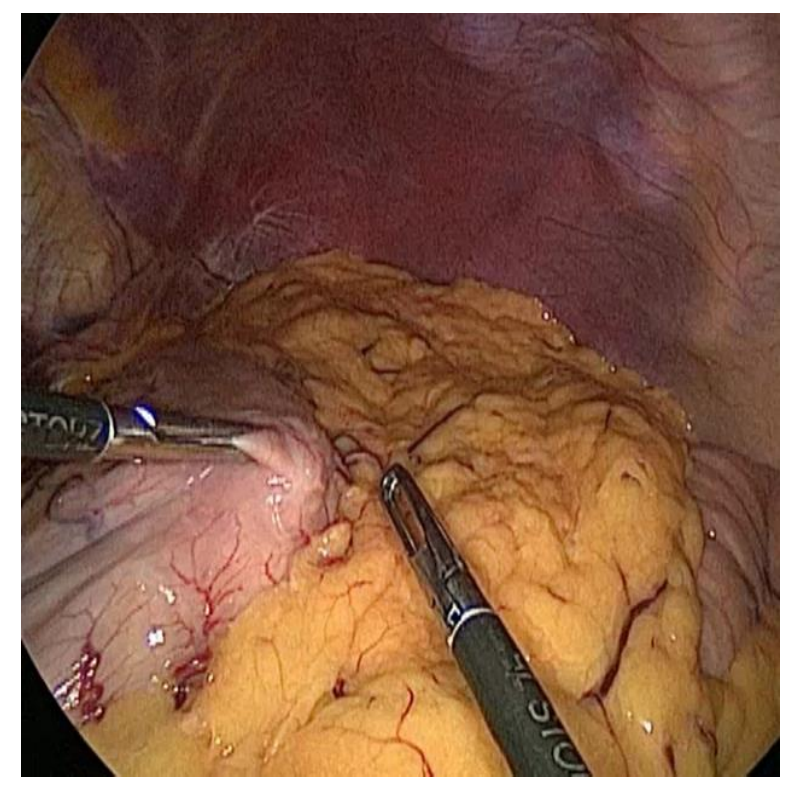

Figure 3 Intraoperative image with the choice of the gastrostomy site.

A purse string using a monofilament resorbable wire is performed. We prefer the monofilament stiches for this step of the surgery for the capacity to secure easily the tube in place. The diameter of the purse string is of $1 \mathrm{~cm}$, to allow the gastrotomy. When performing gastrotomy with the electric hook there are two important aspects: cautions must be taken to not fragilize the stitch during the electrocoagulation of the gastric wall and is also important to well visualise the gastric lumen and to avoid the placement of the tube between the layers of the gastric wall. (Figure 4,5)

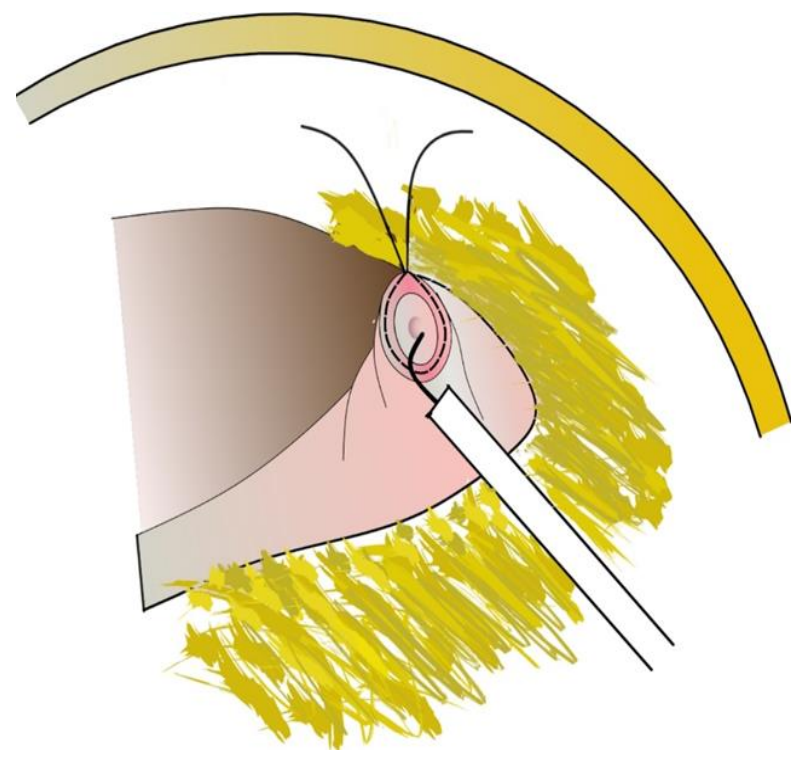

Figure 4 The gastrotomy is performed inside the purse-string. 


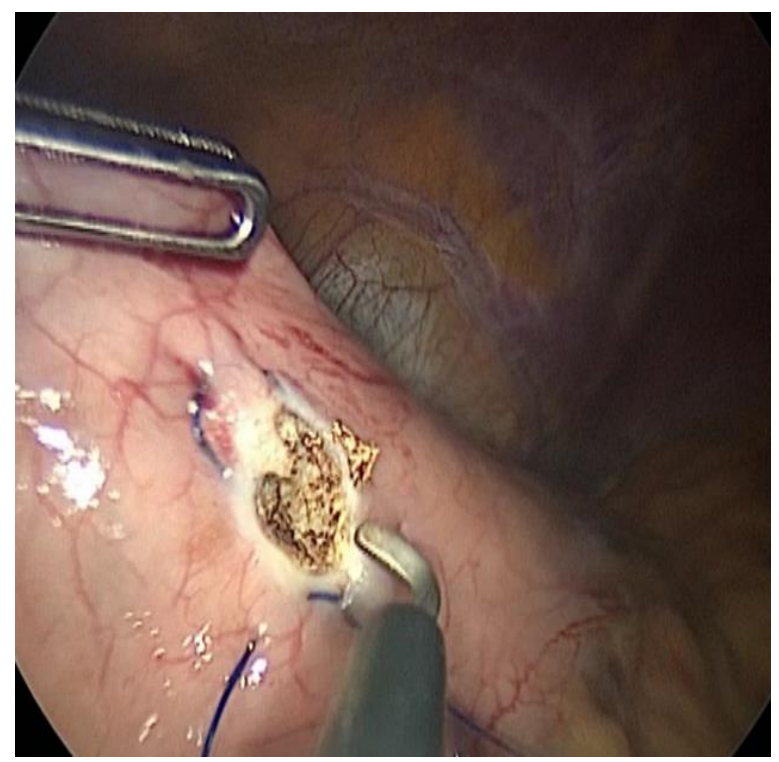

Figure 5 Intraoperative image of the gastrotomy.

\section{Feeding tube introduction}

A place is chosen on the anterior abdominal wall for the placement of the feeding tube. For this step of the surgery a $5 \mathrm{~mm}$ trocar is placed in the left epigastric area. The site must respect few principles: should not be close to the costal grill and must represent the shortest trajectory between the gastrotomy site and skin incision, in order to avoid too much tension on the gastric wall. The feeding tube balloon is tested before the introduction by the instillation of air or saline solution depending of the feeding tube instructions. The tube can be introduced by the trocar or by the trocar parietal incision. Care must be taken to not injured the balloon during the introduction. The tube is placed via the gastrostomy in the gastric lumen and the balloon is inflate. The purse string is closed and well secured around the tube. The tube is tested by injecting saline solution, to test the permeability and the sealing around the tube. (Figure 6, 7)

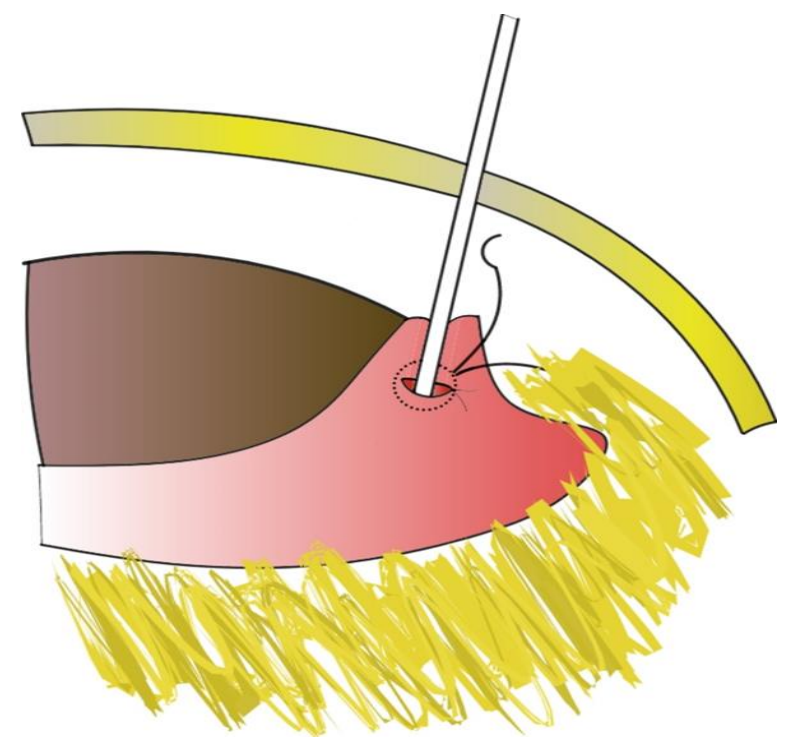

Figure 6 The feeding tube introduction into the stomach lumen. 


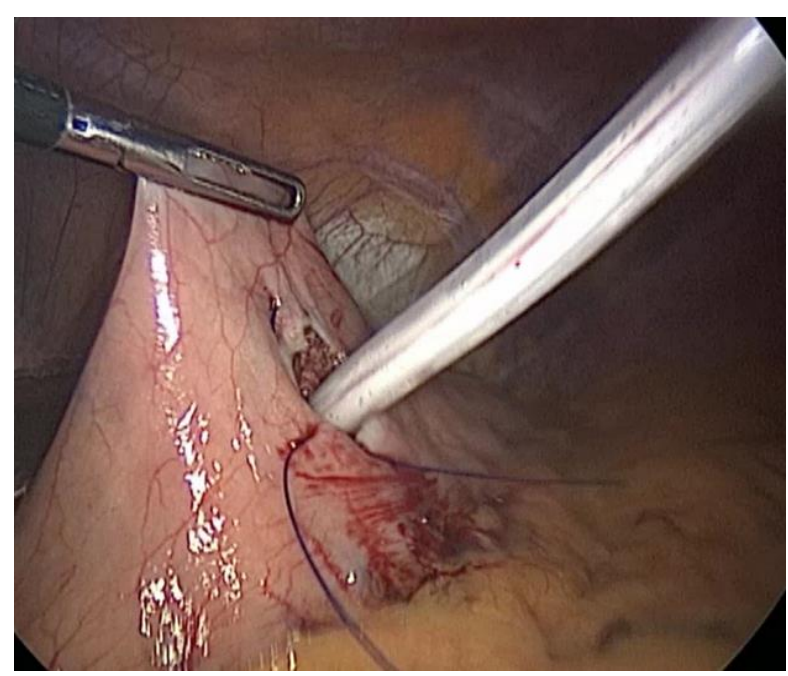

Figure 7 Intraoperative image of the feeding tube introduction into the stomach lumen.

A second purse string is performed using the same suture. This suture is performed on the serosa layer and a special attention must be taken to not injure the tube or the balloon. This suture is placed at $1 \mathrm{~cm}$ from the previous to insure a good invagination of the first suture. The purse string is closed and the serosa vertical tunnel, which will provide a good sealing around the tube is performed.

\section{Parietal fixation}

For the fixation of the gastric wall to the abdominal wall we use resorbable braided thread like Vycril 2.0. Usually two simple stiches are used for the fixation of the stomach to the abdominal wall. If there is too much traction on the stomach, then the pressure of the pneumoperitoneum is reduced and the assistant can press on the abdominal wall. There are multiple mechanisms who will assure the good sealing of the tube as the balloon, the purse string and the parietal fixation of the gastric wall. At the final pf the procedure the permeability of the tube is retested by reinjecting $50 \mathrm{ml}$ of saline solution. (Figure 8, 9)

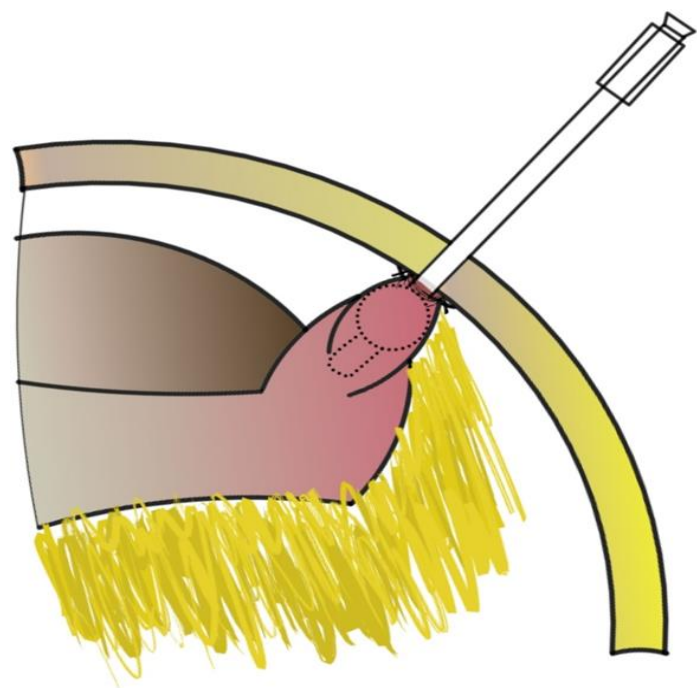

Figure 8 Final view. 


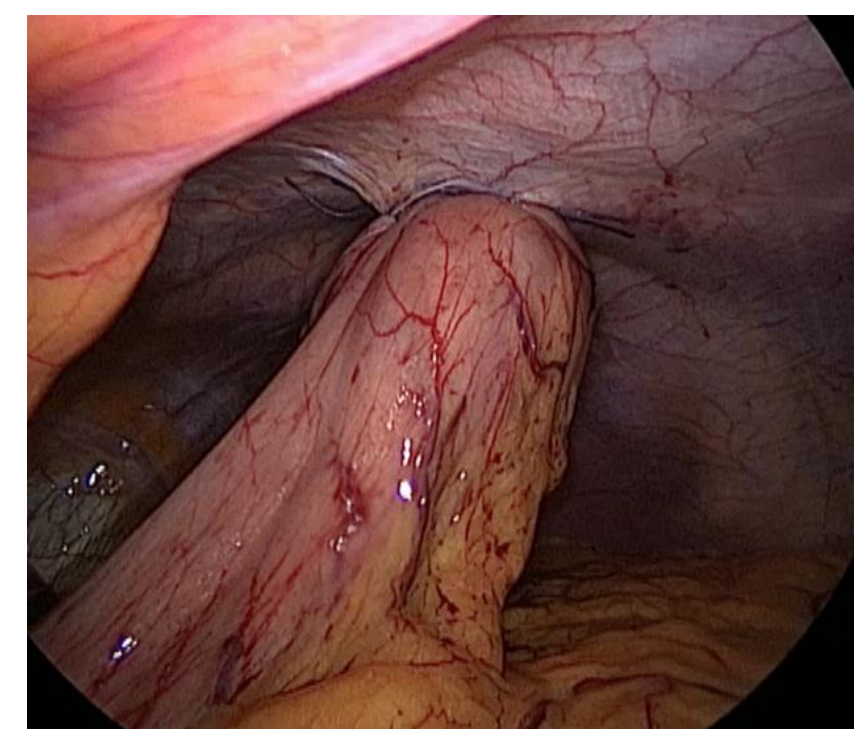

Figure 9 Intraoperative final view.

\section{Justification of the technique}

We propose a solution for totally laparoscopic feeding tube gastrostomy placement. There are another surgical techniques as partial laparoscopic placement or tube witzelisation, who will be discussed separately. This technique respect the principles of Stamm technique, with modification related to the laparoscopic approach. We propose the placement of the surgical team at the right side of the patient, in French position and not in American position, between the legs of the patient, due to the better ergonomics during the fixation of the tube on the abdominal wall. In case of the right trocar is placed to the left side, as in the flank, the instrument might be too tangential to the abdominal wall, and make the suture more difficult during the stomach fixation to the abdominal wall. The landmark of the trocars placement must be adapted to the patient anatomy, but the basic principles of laparoscopy as the triangulation of the trocars must be respected. Concerning the sutures, for the parietal fixation we prefer to use braided resorbable sutures, because the first knot is done under traction. If there is some traction during the laparoscopy on the stomach, once the abdomen is deflated the level of traction will significantly ameliorate.

\section{Conclusion}

Totally laparoscopic feeding gastrostomy can be a routine operation for the experienced surgeons, especially those with experience in laparoscopic surgery. If the gesture is not optimal, the surgery can be a source of postoperative complications. This surgery can be standardised due to the regular anatomy and the simplicity of the gestures.

\section{Compliance with ethical standards}

\section{Disclosure of conflict of interest}

The authors declare that there is no conflict of interest .

\section{References}

[1] Anselmo CB, Tercioti Junior V, Lopes LR, Coelho Neto J de S, Andreollo NA. Gastrostomia cirúrgica: indicações atuais e complicações em pacientes de um hospital universitário. Rev Col Bras Cir. Dec 2013; 40(6): 458-62.

[2] Strong AT, Ponsky JL. Following the light: A history of the percutaneous endoscopic gastrostomy tube. $2017 ; 4$.

[3] Souza EC. SURGICAL GASTROSTOMY BASED ON ENDOSCOPIC CONCEPTS. Arq Bras Cir Dig. 2016; 29(1): 50-52.

[4] George R, Radhakrishna V, Mathew M, Thenamangalath A, Rahman A. Modified Hasson technique: a quick and safe entry of first port into the abdomen. Int Surg J. 25 Jul 2019; 6(8): 2802. 